\title{
REGENERAÇÃO PÓS-FOGO EM UM FRAGMENTO DE FLORESTA ESTACIONAL SEMIDECIDUAL NO MUNICÍPIO DE VIÇOSA, MG
}

\section{POST-FIRE REGENERATION IN A SEMIDECIDUOUS MESOPHYTIC FOREST FRAGMENT IN THE COUNTY OF VIÇOSA, MG}

\author{
Sebastião Venâncio Martins ${ }^{1}$ Guido Assunção Ribeiro ${ }^{1}$ \\ Wilson Marcelo da Silva Junior ${ }^{2}$ Mauro Eloi Nappo ${ }^{3}$
}

\begin{abstract}
RESUMO
Estudou-se a vegetação colonizadora seis meses após perturbação por fogo, em uma área do Campus da Universidade Federal de Viçosa, em Viçosa $\left(20^{\circ} 45^{\prime} \mathrm{S}, 42^{\circ} 51^{\prime} \mathrm{W}\right)$, MG. A área total de floresta secundária atingida por incêndio foi de 12 hectares, apresentando níveis diferenciados de destruição da vegetação. $\mathrm{O}$ estudo concentrou-se no trecho onde o efeito do fogo foi mais drástico, com a destruição total da cobertura vegetal. Dentro da área de estudo, foram comparadas duas toposseqüências de relevo: côncavo (ravina) e convexo (crista). Em cada toposseqüência, foram instaladas dezoito parcelas contíguas de $5 \times 5 \mathrm{~m}$, totalizando 36 parcelas, nas quais foram identificadas todas as plantas vasculares. No total, foram amostradas 75 espécies, pertencentes a 26 famílias. As famílias mais ricas em espécies foram: Asteraceae (14), Poaceae (10), Malvaceae (7) e Fabaceae (6). Na toposseqüência crista as espécies com maior valor de cobertura foram: Sida carpinifolia seguida de Melinis minutiflora, Diodia alata e Eupatorium laevigatum, e na ravina: Melinis minutiflora, Eupatorium laevigatum, Sida carpinifolia e Sidastrum paniculatum. Todas as parcelas apresentaram mais de $90 \%$ da área coberta por vegetação herbácea e subarbustiva, destacando a importância dessa vegetação inicial na proteção do solo contra processos erosivos em relevos acidentados.
\end{abstract}

Palavras-chave: Regeneração natural, ecologia do fogo, degradação florestal, floresta tropical.

\begin{abstract}
The colonizer vegetation was studied six months after fire disturbance in an area of the Campus of the Universidade Federal de Viçosa $\left(20^{\circ} 45^{\prime} \mathrm{S}, 42^{\circ} 51^{\prime} \mathrm{W}\right)$, MG. The total burned area of the secondary forest was of 12 ha presenting differentiated levels of vegetation destruction. The study was concentrated in the space where the effect from fire was more drastic, and a total destruction of the vegetative cover occurred. Within the study area, two relief toposequences were compared: concave (ravine) and convex (ridge). In each toposequence, 18 contiguous plots $(5 \times 5 \mathrm{~m})$ were installed, totalizing 36 plots, where all vascular plants were identified. A total of 75 species pertaining to 26 families were sampled. In relation to the species, the richest ones were: Asteraceae (14), Poaceae (10), Malvaceae (7) and Fabaceae (6). In the toposequence ridge, the species showing higher soil covering values were Sida carpinifolia followed by Melinis minutiflora, Diodia alata and Eupatorium laevigatum, while in ravine they were Melinis minutiflora, Eupatorium laevigatum, Sida carpinifolia and Sidastrum paniculatum. All plots presented more than $90 \%$ of the area covered by herbaceous and subshrubby vegetation, so emphasizing the importance of this initial vegetation in protecting the soil against erosion processes on uneven reliefs.
\end{abstract}

Key words: Regeneration, fire ecology, forest degradation, tropical forest.

\section{INTRODUÇÃO}

Ecossistemas e paisagens são alterados no tempo e no espaço em razão de características da vegetação e regimes de distúrbios. Interações entre eventos de distúrbios e a sucessão vegetal criam o mosaico florestal, onde manchas ou eco-unidades da floresta tendem a apresentar composições florísticas e estruturas diferenciadas (Oldeman, 1983; Clark, 1990; Camp et al., 1996).

1. Engenheiro Florestal, Dr., Professor do Departamento de Engenharia Florestal, Universidade Federal de Viçosa, CEP 36571-000, Viçosa (MG).

2. Biólogo, Mestrando em Ciência Florestal, Departamento de Engenharia Florestal, Universidade Federal de Viçosa, CEP 36571-000, Viçosa (MG).

3. Engenheiro Florestal, Doutorando em Ciência Florestal, Departamento de Engenharia Florestal, Universidade Federal de Viçosa, CEP 36571-000, Viçosa (MG). 
Diversos tipos de distúrbios naturais ou antrópicos podem alterar a dinâmica da vegetação florestal e desencadear o processo de sucessão secundária, como abertura de clareiras naturais pela queda de árvores, deslizamentos de terra, atividades vulcânicas, ataque de insetos e incêndios florestais. Dentre essas fontes de perturbação, vários autores relatam a importância do fogo, por afetar o funcionamento dos ecossistemas, influenciando a sucessão vegetal, a composição e estrutura florestal, o acúmulo de serapilheira, a ciclagem de nutrientes, a população de insetos, o banco de sementes no solo e a rebrota de espécies arbustivo-arbóreas (Uhl et al., 1981; Kauffman, 1991; Mutch e Cook, 1996; Cochrane e Schulze, 1999).

Em áreas com relevo acidentado, os efeitos do fogo podem ser ainda mais drásticos, uma vez que a queima causando a eliminação da cobertura vegetal favorece a perda de nutrientes por escorrimento superficial e lixiviação (Sanches, 1981; Coutinho, 1990; Martins et al., 1995), além de tornar a superfície do solo desprotegida, intensificando a ocorrência de processos erosivos (Barro e Conard, 1987). Assim, fatores como declividade do terreno, tempo de exposição do solo, intensidade do fogo, proximidade de fontes de propágulos, componente florístico remanescente na forma de sementes ou tecidos vegetais com capacidade de rebrotar irão determinar a seqüência florística e a velocidade da regeneração (Uhl et al., 1982; Castellani e Stubblebine, 1993; Penha, 1998).

Nesse contexto, o estudo das comunidades colonizadoras iniciais de áreas onde a vegetação florestal foi destruída pelo fogo é de grande relevância, uma vez que a cobertura inicial do solo por espécies herbáceas e subarbustivas pode proteger o solo (Pausas et al., 1999) e gerar condições ecológicas mais favoráveis ao estabelecimento de espécies arbustivas e arbóreas. Por outro lado, essas comunidades invasoras, quando muito agressivas, podem intensificar a competi ção por recursos e, seguindo um modelo de inibição, atrasar o processo de regeneração (Connell e Slatyer, 1977; Harper, 1977). Assim, o conhecimento florístico e ecológico, dessa vegetação inicial, pode auxiliar programas de seleção e manejo de espécies, visando à restauração de áreas degradadas (Brown e Amacher, 1999).

Este estudo teve por objetivos: conhecer a composição florística da vegetação colonizadora de um fragmento de floresta estacional semidecidual perturbada por fogo, em duas toposseqüências de relevo (côncavo e convexo) e analisar a representatividade das espécies amostradas.

\section{MATERIAL E MÉTODOS}

$\mathrm{O}$ estudo foi realizado em uma área de floresta estacional semidecidual secundária, situada no Campus da Universidade Federal de Viçosa ( $20^{\circ} 45^{\prime}$ S, $42^{\circ} 51^{\prime}$ W), Viçosa, MG.

O clima da região é do tipo $\mathrm{CWb}$, pela classificação de Köeppen, ou seja tropical de altitude, caracterizado por verões quentes e chuvosos e invernos frios e secos, com precipita ção média anual de 1.221 $\mathrm{mm}$, e temperatura média anual de $19^{\circ} \mathrm{C}$ (Golfari, 1975; Brasil, 1992). O relevo é caracterizado por colinas alinhadas em forma de espigões, bastante seccionadas pela rede de drenagem, com altitudes variando entre 650 e 800 m. Predominam na região os solos do tipo Latossolo Vermelho Amarelo distrófico e Podzólico Vermelho Amarelo câmbico (Arruda et al., 1999).

Um incêndio ocorrido em agosto de 1999, destruiu 12 hectares da vegetação nativa, apresentando níveis diferenciados de destruição. O estudo concentrou-se no trecho onde o efeito do fogo foi mais drástico, com destruição total da cobertura vegetal.

Dentro da área de estudo, foram comparadas floristicamente, por meio do índice de Jaccard, duas toposseqüências de relevo: côncavo (ravina) e convexo (crista). Em cada toposseqüência, foram instaladas frzoito parcelas contíguas de $5 \times 5 \mathrm{~m}$, totalizando 36 parcelas nas quais foram coletadas e identificadas todas as espécies vasculares.

Determinaram-se, para as espécies amostradas nas duas toposseqüências, os parâmetros: valor de cobertura (Braun-Blanquet, 1979) e freqüência absoluta (Mueller-Dombois e Ellemberg, 1974). A determinação da cobertura das espécies relaciona-se com a escala de valores médios da superficíe coberta por estas. O grau de cobertura por parcela (de $5 \times 5 \mathrm{~m}$ ) foi determinado mediante avaliação visual, em porcentagem $(0-100 \%)$, da área da parcela coberta por cada espécie (Rondon Neto, 1999). 
O valor de cobertura foi calculado segundo a express ão abaixo:

$\mathrm{Vc}_{\mathrm{i}}=\sum_{i=1}^{n} \frac{C i}{n}$

Em que:

Vci $=$ valor de cobertura da $\mathrm{i}$-ésima espécie, em $\% ; C i=$ porcentagens de cobertura da $\mathrm{i}$-ésima espécie; $n=$ número total de parcelas amostradas.

A freqüência absoluta de cada espécie foi obtida pela expressão:

$\mathrm{FAi}=\frac{U i}{U t} \times 100$

Em que:

$\mathrm{FAi}=$ Freqüência absoluta da i-ésima espécie, em \%; $U i=$ Número de unidades amostrais em que a espécie $i$ foi amostrada; $U t=$ Número total de unidades amostrais.

Avaliou-se a ocorrência de rebrota nas espécies amostradas nas parcelas. A constatação e identificação das espécies, que se estabeleceram com base na rebrota de cepas, foram realizadas examinando--se, em cada indivíduo, a inserção das brotações na base do caule. Já para espécies com rebrota de raízes gemíferas, foram realizadas escavações e exposição do sistema radicular partindo da base do caule dos brotos emitidos, a fim de confirmar sua ligação com os troncos dos indivíduos adultos (Penha, 1998) que foram atingidos pelo fogo.

Foi estimada a biomassa epígea seca para as duas toposseqüências pelo método destrutivo, ou seja por meio do corte ao nível do solo de todas as plantas presentes no interior de dezoito parcelas de $0,5 \times 0,5$ $\mathrm{m}$ em cada toposseqüência, lançadas sistematicamente (amostragem sistemática) em intervalos de $5 \mathrm{~m}$, do lado externo de cada parcela do levantamento florístico. O material foi acondicionado em sacos plásticos etiquetados e conduzidos ao laboratório, onde foi seco em estufa a $75^{\circ} \mathrm{C}$, até peso constante. Com os valores de biomassa epígea seca obtidos em cada uma das 18 parcelas e transformados em Kg.ha ${ }^{-1}$, obteve-se a biomassa epígea seca média por toposseqüência, ou seja a média de dezoito repetições. Compararam-se então, as médias de biomassa epígea seca entre as duas toposseqüências, pelo teste-t para dados independentes, com nível de probabilidade de 0,05 .

\section{RESULTADOS E DISCUSSÃO}

Considerando as duas toposseqüências (crista e ravina), foram amostradas 75 espécies, pertencentes a 26 famílias. Dessas, 63 foram identificadas em nível de espécie, dez em nível de gênero e duas em nível de família (Tabela 1). As famílias com maior riqueza específica foram Asteraceae (14), Poaceae (10), Malvaceae (7) e Fabaceae (6). Essas famílias, representadas por grande número de espécies herbáceas e subarbustivas ruderais ou invasoras daninhas, se destacaram em riqueza em comunidades iniciais da sucessão em outras áreas de floresta estacional semidecidual após fogo (Castellani e Stubblebine, 1993) e desmatamento (Rondon Neto, 1999).

$\mathrm{Na}$ comparação entre as duas toposseqüências, o índice de Jaccard obtido foi de 54,67\%, indicando elevada similaridade florística, uma vez que duas áreas são consideradas floristicamente semelhantes, quando esse índice é superior a 25\% (Mueller-Dombois e Ellenberg, 1974). A biomassa epígea seca (Tabela 2) não diferiu significativamente entre as duas toposseqüências $(P>0,05)$. Essa semelhança florística e de biomassa aérea é explicada pela proximidade das duas áreas e pelo fato de terem sido submetidas ao mesmo tipo e intensidade de distúrbio.

Para as duas toposseqüências, ocorreu predominância de espécies herbáceas e subarbustivas, refletida nos valores de cobertura e de freqüência absoluta (Tabela 1). Na toposseqüência crista, as espécies com maior valor de cobertura foram: Sida carpinifolia, seguida de Melinis minutiflora, Diodia alata e Eupatorium laevigatum, e na ravina: Melinis minutiflora, Eupatorium laevigatum, Sida carpinifolia e 
Sidastrum paniculatum. Tais espécies apresentaram também valores elevados de freqüência absoluta, cabendo ressaltar que Eupatorium laevigatum foi amostrada em todas as parcelas do levantamento e Melinis minutiflora em todas as parcelas da ravina e em $94,44 \%$ da crista. À exceção de Diodia alata, as demais espécies desse grupo dominante se encontravam em estádio reprodutivo no momento do levantamento, sugerindo que poderão permanecer na área e exercer ainda importante papel nas fases iniciais da sucess ão secundária.

TABELA 1: Espécies amostradas após seis meses da ocorrência de incêndio em fragmento de floresta estacional semidecidual no município de Viçosa, MG.

\begin{tabular}{|c|c|c|c|c|c|c|}
\hline \multirow{2}{*}{$\begin{array}{l}\text { FAMÍLIAS } \\
\text { Espécies }\end{array}$} & \multirow[b]{2}{*}{ Hábito } & \multirow[b]{2}{*}{ Rebrota } & \multicolumn{2}{|c|}{ Valor de cobertura } & \multicolumn{2}{|c|}{ Freqüência } \\
\hline & & & $\mathrm{Cr}$ & $\mathrm{Rv}$ & $\mathrm{Cr}$ & $\mathrm{Rv}$ \\
\hline \multicolumn{7}{|l|}{ ARISTOLOCHIACEAE } \\
\hline Aristolochia sp. & $\mathrm{T}$ & & 0,15 & & 11,11 & \\
\hline \multicolumn{7}{|l|}{ ASTERACEAE } \\
\hline Acanthospermum australe (Loef.) O.Kuntze & $\mathrm{E}$ & & 0,17 & & 11,11 & \\
\hline Agerantum conysoides $\mathrm{L}$. & $\mathrm{E}$ & & 0,28 & 0,78 & 22,22 & 61,11 \\
\hline Ambrosia polystachya DC. & A & & & 0,10 & & 11,11 \\
\hline Bidens sp. & E & & 0,1 & 1,83 & 5,56 & 33,33 \\
\hline Elephamtopus mollis H.B.K. & $\mathrm{E}$ & & 0,17 & & 5,56 & \\
\hline Elvira biflora (L.) DC. & $\mathrm{E}$ & & & 0,1 & & 5,56 \\
\hline Emilia sonchifolia DC. & E & & 0,15 & & 5,56 & \\
\hline Eupatorium laevigatum Lam. & A & & 6,78 & 11,81 & 100,00 & 100,00 \\
\hline \multicolumn{7}{|l|}{ ASTERACEAE } \\
\hline Eupatorium sp. & SA & & 0,30 & & 16,7 & \\
\hline Eupatorium squalidum DC. & SA & & 4,08 & 0,72 & 72,22 & 16,67 \\
\hline Piptocarpha macropoda Baker & AR & $\mathrm{x}$ & & 0,11 & & 11,11 \\
\hline Vernonia cognata Less. & SA & & 0,36 & 0,17 & 38,89 & 22,22 \\
\hline Vernonia polyanthes Less. & A & $\mathrm{x}$ & 0,69 & 1,03 & 72,22 & 50,00 \\
\hline Vernonia sp. & SA & & 0,20 & 0,21 & 11,11 & 5,56 \\
\hline \multicolumn{7}{|l|}{ APOCYNACEAE } \\
\hline Hymatanthus phagedaenicus (Mart.)R.E. & AR & $\mathrm{x}$ & 0,28 & 0,20 & 5,56 & 5,56 \\
\hline Peschiera sp. & $\mathrm{A}$ & & & 0,12 & & 5,56 \\
\hline \multicolumn{7}{|l|}{ BIGNONIACEAE } \\
\hline Jacaranda macrantha Cham. & AR & $\mathrm{x}$ & 0,57 & & 5,56 & \\
\hline Pyrostegia venusta Miers. & $\mathrm{T}$ & $\mathrm{x}$ & 0,72 & 1,19 & 72,22 & 61,11 \\
\hline Bignoniaceae 1 & $\mathrm{~T}$ & $\mathrm{x}$ & 0,90 & 0,83 & 16,67 & 72,22 \\
\hline \multicolumn{7}{|l|}{ CAESALPINIACEAE } \\
\hline Chamaecrista nictitans (L.) Moench & SA & & 0,86 & 0,28 & 55,56 & 27,78 \\
\hline Chamaecrista rotundifolia (Pers.) Greene & SA & & 0,20 & 0,10 & 11,11 & 11,11 \\
\hline Senna obtusifolia (L.) Irwin \& Barnery & SA & & 0,15 & 0,14 & 16,67 & 16,67 \\
\hline \multicolumn{7}{|l|}{ CECROPIACEAE } \\
\hline Cecropia hololeuca Miq. & $\mathrm{AR}$ & $\mathrm{x}$ & & 0,28 & & 5,56 \\
\hline \multicolumn{7}{|l|}{ COMMELINACEAE } \\
\hline Commelina erecta $\mathrm{L}$. & $\mathrm{E}$ & & 0,1 & 0,50 & 11,11 & 55,56 \\
\hline \multicolumn{7}{|l|}{ CYPERACEAE } \\
\hline Scleria pterota Presl. & $\mathrm{E}$ & & & 0,11 & & 16,67 \\
\hline \multicolumn{7}{|l|}{ ERYTHROXYLACEAE } \\
\hline Erythroxylum pelleterianum St.Hil. & A & $\mathrm{x}$ & & 0,28 & & 16,67 \\
\hline
\end{tabular}


TABELA 1: Continuação ...

\begin{tabular}{|c|c|c|c|c|c|c|}
\hline \multirow{2}{*}{$\begin{array}{l}\text { FAMíLIAS } \\
\text { Espécies }\end{array}$} & \multirow[b]{2}{*}{ Hábito } & \multirow[b]{2}{*}{ Rebrota } & \multicolumn{2}{|c|}{ Valor de cobertura } & \multicolumn{2}{|c|}{ Freqüência } \\
\hline & & & $\mathrm{Cr}$ & $\mathrm{Rv}$ & $\mathrm{Cr}$ & $\mathrm{Rv}$ \\
\hline \multicolumn{7}{|l|}{ EUPHORBIACEAE } \\
\hline Alchornea triplinervea (Spr.) Muell. Arg. & $\mathrm{AR}$ & $\mathrm{x}$ & & 0,56 & & 5,56 \\
\hline Croton glandulosus $\mathrm{L}$. & SA & & & 0,22 & & 11,11 \\
\hline Mabea fistulifera Mart. & AR & $\mathrm{x}$ & 2,1 & 5,77 & 33,33 & 38,89 \\
\hline \multicolumn{7}{|l|}{ FABACEAE } \\
\hline Aeschynomene falcata (Poir.) DC. & $A S$ & & 0,58 & & 11,11 & \\
\hline Dalbergia nigra Allen. Ex Benth. & $\mathrm{AR}$ & $\mathrm{x}$ & 1,69 & 2,11 & 38,89 & 55,56 \\
\hline Desmodium barbatum (L.) Benth. & $\mathrm{E}$ & & & 0,33 & & 11,11 \\
\hline \multicolumn{7}{|l|}{ FABACEAE } \\
\hline Machaerium stipitatum (DC.) Vog. & AR & $\mathrm{x}$ & & 0,19 & & 5,56 \\
\hline Platypodium elegans Vog. & AR & $\mathrm{x}$ & 0,56 & & 5,56 & \\
\hline Stylosanthes viscosa $\mathrm{Sw}$. & AS & & & 0,14 & & 11,11 \\
\hline \multicolumn{7}{|l|}{ FLACOURTIACEAE } \\
\hline Casearia sylvestris $\mathrm{Sw}$. & $\mathrm{AR}$ & $\mathrm{x}$ & & 0,12 & & 5,56 \\
\hline \multicolumn{7}{|l|}{ LAMIACEAE } \\
\hline Hyptis suaveolens (L.) Poit. & AS & & 0,1 & 0,61 & 5,56 & 27,78 \\
\hline \multicolumn{7}{|l|}{ MALVACEAE } \\
\hline Sida carpinofolia $\mathrm{L}$. & AS & & 27,83 & 6,46 & 94,44 & 61,11 \\
\hline Sida glaziovii K. Schum. & AS & & 0,95 & 0,89 & 5,56 & 27,78 \\
\hline Sida linifolia Cav. & AS & & 1,72 & 1,29 & 100,00 & 66,67 \\
\hline Sida urens L. & AS & & 0,32 & 0,15 & 27,78 & 11,11 \\
\hline Sidastrum micranthum (St.Hil.) Fryxell & AS & & 0,1 & 0,28 & 11,11 & 11,11 \\
\hline Sidastrum paniculatum (L.) Fryxell & AS & & 2,81 & 3,47 & 88,89 & 50,00 \\
\hline Malvaceae 1 & AS & & & 0,11 & & 5,56 \\
\hline \multicolumn{7}{|l|}{ MELASTOMATACEAE } \\
\hline Leandra nianga Cong. & A & & & 0,14 & & 16,67 \\
\hline \multicolumn{7}{|l|}{ MENISPERMACEAE } \\
\hline Cissampelos sp. & $\mathrm{T}$ & & 0,16 & 0,11 & 11,11 & 22,22 \\
\hline \multicolumn{7}{|l|}{ MIMOSACEAE } \\
\hline Anadenanthera peregrina Speg. & AR & $\mathrm{x}$ & 0,1 & 0,11 & 5,56 & 11,11 \\
\hline Mimosa pigra $\mathrm{L}$. & AS & & 0,83 & & 16,67 & \\
\hline Mimosa sp. & AS & & 2,5 & 0,19 & 16,67 & 22,22 \\
\hline Piptadenia gonoacantha (Mart.) Macbr. & $\mathrm{AR}$ & $\mathrm{x}$ & 0,56 & 0,17 & 5,56 & 5,56 \\
\hline \multicolumn{7}{|l|}{ PHYTOLACCACEAE } \\
\hline Phytolacca thyrsiflora Fenzl. & A & $\mathrm{x}$ & 0,16 & & 11,11 & \\
\hline \multicolumn{7}{|l|}{ POACEAE } \\
\hline Andropogon bicornis $\mathrm{L}$. & $\mathrm{E}$ & & 0,11 & 0,28 & 22,22 & 33,33 \\
\hline Brachiaria decumbens Stapf. & $\mathrm{E}$ & & & 0,56 & & 5,56 \\
\hline Digitaria insularis (L.) Fedde & $\mathrm{E}$ & & & 0,22 & & 16,67 \\
\hline Eragrostis sp. & $\mathrm{E}$ & & 0,47 & 0,64 & 50,00 & 33,33 \\
\hline Hyparrhenia rufa (Ness.) Stapf. & E & & & 0,28 & & 5,56 \\
\hline Imperata brasiliensis Trin. & $\mathrm{E}$ & $\mathrm{x}$ & 1,47 & 2,28 & 27,78 & 22,22 \\
\hline
\end{tabular}




\begin{tabular}{lllllll} 
Melinis minutiflora Beauv. & E & X & 26,42 & 39,84 & 94,44 & 100,00 \\
\hline & & & & & & Continua $\ldots$
\end{tabular}

TABELA 1: Continuação ...

\begin{tabular}{|c|c|c|c|c|c|c|}
\hline \multirow{2}{*}{$\begin{array}{l}\text { FAMÍLIAS } \\
\text { Espécies }\end{array}$} & \multirow[b]{2}{*}{ Hábito } & \multirow[b]{2}{*}{ Rebrota } & \multicolumn{2}{|c|}{ Valor de cobertura } & \multicolumn{2}{|c|}{ Freqüência } \\
\hline & & & $\mathrm{Cr}$ & $\mathrm{Rv}$ & $\mathrm{Cr}$ & $\mathrm{Rv}$ \\
\hline \multicolumn{7}{|l|}{ POACEAE } \\
\hline Panicum maximum Ness. & $\mathrm{E}$ & & 0,42 & 0,19 & 55,56 & 27,78 \\
\hline Panicum ripens $\mathrm{L}$. & $\mathrm{E}$ & & 0,22 & 0,12 & 16,67 & 11,11 \\
\hline Setaria geniculata (Lam.) Beauv. & $\mathrm{E}$ & & 0,11 & 0,28 & 11,11 & 5,56 \\
\hline \multicolumn{7}{|l|}{ POLYGALACEAE } \\
\hline Polygala violaceae $\mathrm{L}$. & AS & & 0,11 & 0,10 & 5,56 & 5,56 \\
\hline \multicolumn{7}{|l|}{ RUBIACEAE } \\
\hline Diodia alata Nees \& Mart. & AS & & 7,00 & 3,03 & 100,00 & 38,89 \\
\hline Spermacoce capitata Ruiz \& Pav. & AS & & & 0,14 & & 16,67 \\
\hline Spermacoce verticillata $\mathrm{L}$. & AS & & 0,22 & 0,18 & 22,22 & \\
\hline \multicolumn{7}{|l|}{ RUTACEAE } \\
\hline Zanthoxylum rhoifolium Lam. & $\mathrm{AR}$ & $\mathrm{x}$ & 0,48 & 0,53 & 5,56 & 5,56 \\
\hline \multicolumn{7}{|l|}{ SAPINDACEAE } \\
\hline Serjanea sp. & $\mathrm{T}$ & $\mathrm{x}$ & & 1,19 & & 22,22 \\
\hline \multicolumn{7}{|l|}{ SOLANACEAE } \\
\hline Brunfelsa brasiliensis Benth. & SA & & & 0,10 & & 5,56 \\
\hline Solanum aculeatissimum Jacq. & SA & & & 0,19 & & 11,11 \\
\hline \multicolumn{7}{|l|}{ TILIACEAE } \\
\hline Luehea speciosa Wild. & A & $\mathrm{x}$ & & 0,50 & & 5,56 \\
\hline Triunfetta bartramia L. & SA & & 0,34 & 2,42 & 44,44 & 72,22 \\
\hline \multicolumn{7}{|l|}{ VERBENACEAE } \\
\hline Aegiphila sellowiana Cham. & AR & $\mathrm{x}$ & 1,61 & 2,19 & 22,22 & 33,33 \\
\hline Lantana camara $\mathrm{L}$. & SA & & 0,13 & 0,42 & 5,56 & 22,22 \\
\hline Vitex sp. & A & $\mathrm{x}$ & 0,50 & & 16,67 & \\
\hline
\end{tabular}

Em que: Hábito de vida: $\mathrm{E}=$ erva; $\mathrm{SA}=$ subarbusto; $\mathrm{A}=$ arbusto; $\mathrm{AR}=$ árvore; $\mathrm{T}=$ trepadeira; Toposseqüência do relevo: $\mathrm{Cr}=$ crista; $\mathrm{Rv}=$ ravina; Origem: $\mathrm{Nt}=$ nativa; $\mathrm{Ex}=$ exótica. Estão assinaladas as espécies que se estabeleceram por rebrota.

TABELA 2: Biomassa epígea seca estimada para as toposseqüências crista e ravina, após seis meses da ocorrência de incêndio em fragmento de floresta estacional semidecidual no município de Viçosa, MG.

\begin{tabular}{lc}
\hline Toposseqüência & Biomassa epígea seca $\left(\mathrm{Kg}^{-1}{ }^{-1}\right)$ \\
\hline Crista & $2.814,08 \mathrm{a}$ \\
Ravina & $3.414,25 \mathrm{a}$ \\
\hline
\end{tabular}

Médias seguidas da mesma letra não diferem estatisticamente entre si $(P>0,05)$, pelo teste-t para dados independentes.

Outras espécies como Sida linifolia, Vernonia polyanthes e Pyrostegia venusta, todas com baixos valores de cobertura, apresentaram freqüências elevadas. Sida linifolia encontrava-se em estádio reprodutivo na ocasião do estudo, tendo sido representada por indivíduos isolados ou em pequenos grupos e, portanto, com baixo valor de cobertura tanto na ravina como na crista. Como colonizadora inicial e com baixa agressividade, essa espécie tende a desaparecer ou ter sua população reduzida à medida que a sucessão avance. Já a arbustiva Vernonia polyanthes e a trepadeira Pyrostegia venusta devem ainda destacar-se ao longo das futuras fases iniciais da sucessão, considerando que foram representadas exclusivamente por indivíduos jovens oriundos de rebrota. 
Esse padrão de colonização inicial por espécies herbáceaes e subarbustivas ruderais e invasoras pode ser atribuído a uma série de fatores como a ampla distribuição geográfica desse grupo de plantas, podendo resultar em ampla tolerância ecológica dessas espécies a fatores ambientais, a ocorrência de espécie pantropicais (Gomez-Pompa, 1971; Castellani e Stubblebine, 1993) e a proximidade de áreas com experimentos agrícolas e pastagens que, aliada à síndrome de dispersão predominantemente anemocórica, facilitaria a chegada de sementes.

A rebrota de espécies arbustivas e de trepadeiras típicas de áreas abertas, corrobora a observação de Castellani e Stubblebine (1993), de que tais espécies já estariam presentes antes do distúrbio por fogo, como resultado de outras fontes de perturbações naturais ou antrópicas, como a presença de clareiras e trilhas.

O destaque de Melinis minutiflora nas duas toposseqüências e a riqueza específica elevada da família Poaceae condiz com a agressividade de espécies de gramineas na colonização de grandes áreas abertas, via propagação clonal ou por sementes (Bullock et al., 1995; Miranda e Klink, 1996a,b; Kotanen, 1997). Em áreas de Cerrado, a rebrota de gramineas é um fator importante na regeneração pós- -fogo, conferindo valores elevados de biomassa epígea para esse grupo de plantas (Rosa, 1990; Miranda e Klink, 1996b; Ramos-Neto e Pinheiro-Machado, 1996). Com a destruição da vegetação arbustivo-arbórea pelo fogo, espécies de gramineas são favorecidas pelo aumento nos níveis de luz que estimula o perfilhamento (Deregibus et al., 1985) e pelo aumento na disponibilidade de nutrientes (Coutinho, 1990; Martins et al., 1995; Miranda e Klink, 1996b).

As espécies herbáceas e subarbustivas com maior expressividade estão exercendo importante papel na proteção do solo contra processo erosivos, uma vez que todas as parcelas apresentaram cobertura do solo superior a 90\%. Além disso, essas plantas passam a incorporar mat éria orgânica ao solo e a contribuir com a ciclagem de nutrientes, como sugerem os valores de biomassa epígea seca (Tabela 2), podendo fornecer condições ecológicas mais favoráveis ao estabelecimento de outras espécies arbustivas e arbóreas, gerando assim um modelo sucessional de facilitação (Connell e Slatyer, 1977; Bruno, 2000). Por outro lado, a agressividade dessas espécies e, principalmente de Melinis minutiflora, pode inibir a regeneração de espécies mais tardias na sucessão florestal, como sugeriram Zahawi e Augspurger (1999), avaliando a dominância de uma espécie arbustiva em sucessão secundária no Equador. $\mathrm{O}$ modelo sucessional predominante e o comportamento futuro das espécies, nas duas toposseqüências, só serão entendidos pelo monitoramento periódico da área.

Todas as espécies arbóreas amostradas apresentaram rebrota, corroborando estudos sobre a ocorrência dessa forma de propagação para esse grupo de plantas após fogo, em florestas tropicais (Uhl et al., 1982; Kauffman, 1991; Castellani e Stubblebine, 1993; Penha, 1998) e no Cerrado (Armando, 1994; Nascimento, 1996). Entretanto, a representatividade dessas espécies, expressa em valores de cobertura e de freqüência, foi baixa nas duas toposseqüências. A regeneração por rebrota de espécies arbóreas no início da sucessão pós-fogo ocorre, normalmente, com baixa densidade, sendo mais importante para espécies pioneiras, conferindo-lhes uma oportunidade de ocupação rápida do ambiente perturbado (Uhl et al., 1982; Castellani e Stubblebine, 1993; Penha, 1998).

\section{CONCLUSÕES}

Com base nos resultados obtidos, conclui-se que:

As duas toposseqüências (crista e ravina) apresentaram, até o momento, processos de regeneração semelhantes, refletido pela semelhança florística e de biomassa epígea seca.

Houve predominância de espécies herbáceas e subarbustivas que estão exercendo importante papel na proteção do solo contra processos erosivos e na incorporação de matéria orgânica, considerando os elevados valores de cobertura e de biomassa epígea seca.

A agressividade das espécies herbáceas, principalmente do capim-gordura (Melinis minutiflora), pode estar inibindo a regeneração de espécies arbóreas mais tardias na sucessão. Entretanto, para o entendimento do modelo de sucessão predominante, torna-se necessário o monitoramento da vegetação colonizadora. 
A rebrota foi a principal forma de regeneração das espécies arbóreas sendo, portanto, um mecanismo essencial de manutenção da representatividade dessas espécies na área após o distúrbio.

\section{REFERÊNCIAS BIBLIOGRÁFICAS}

ARMANDO, M.S. O impacto do fogo na rebrota de algumas espécies de árvores do Cerrado. 1994. Dissertação (Mestrado) - Universidade de Brasília, Brasília.

ARRUDA, P.R.R; SILVA, E.; COUTO, L. et al. Problemática ambiental da Bacia Hidrográfica do Ribeirão São Bartolomeu, Viçosa-MG. Revista Árvore, Viçosa, v.23, p.49-53, 1999.

BARRO, S.C.; CONARD, S.G. Use of ryegrass seeding as an emergency revegetation measure in Chaparral ecosystems. Berkeley: U.S. Department of Agriculture, Forest Service, 1987.

BRAUN-BLANQUET, J. Fitossociologia: bases para el estudio de las comunidades vegetales. Madrid: H.Blume Ediciones, 1979.

BRASIL. Ministério da Agricultura. Departamento Nacional de Meteorologia. Normais climatológicas (1961-1990). Brasília, 1992. 84p.

BROWN, R.W.; AMACHER, M.C. Selecting plant species for ecological restoration: a perspective for land managers. In: HOLZWORTH, L.K.; BROWN, R.W. (Eds.). Revegetation with native species. Ogden: U.S. Department of Agriculture, Forest Service, 1999. p.1-16.

BRUNO, J.F. Facilitation of cobble beach plant communities through habitat modification by Spartina alterniflora. Ecology, Washington, v.81, n.5, p.1179-1192, 2000.

BULLOCK, J.M.; CLEAR HILL, B.; SILVERTOWN, J. et al. Gap colonization as a source of grassland community change: effects of gap size and grazing on the rate and mode of colonization by different species. Oikos, Copenhagen, v.72, n.2, p.273-282, 1995.

CAMP, A.E.; HESSBURG, P.F.; EVERETT, R.L. Dynamically incorporating late-successional forest in sustainable landscapes. In: HARDY, C.C.; ARNO, S.F. (Eds.). The use of fire in forest restoration. Ogden: U.S. Department of Agriculture, Forest Service, 1996. p.20-23.

CASTELLANI, T.T.; STUBBLEBINE, W.H. Sucessão secundária inicial em mata tropical mesófila, após perturbação por fogo. Revista Brasileira de Botânica, v.16, p.181-203, 1993.

CLARK, D.A. The role of disturbance in the regeneration of neotropical moist forest. In: BAWA, K.S.; HEDLEY, M. (Eds.). Reproductive ecology of tropical forest plants. Paris: Pathernon Publishing Group, UNESCO, 1990. p.291-315.

COCHRANE, M.; SCHULZE, M.D. Fire as a recurrent event in tropical forests of the eastern Amazon: effects on forest structure, biomass, and species composition. Biotropica, Kansas, v.31, n.1, p.2-16, 1999.

CONNEL, J.H.; SLATYER, R.O. Mechanisms of succession in natural communities and their role in community stability and organization. The American Naturalist, Chicago, v.111, n.982, p.1119-1144, 1977.

COUTINHO, L.M. O Cerrado e a ecologia do fogo. Ciência Hoje, Brasília, v.12, p.131-138, 1990.

DEREGIBUS, V.A.; SANCHEZ, R.A.; CASAL, J.J. et al. Tillering responses to enrichment of red light beneath the canopy in a humid natural grassland. Journal of Applied Ecology, London, v.22, p.199-206, 1985.

GOLFARI, L. Zoneamento ecológico do Estado de Minas Gerais. Belo Horizonte: Centro de Pesquisa Florestal da Região do Cerrado, 1975. 65p.

GOMEZ-POMPA, A. Posible papel de la vegetación secundaria en la evolución de la flora tropical. Biotropica, Kansas, v.3, p.125-135, 1971.

HARPER, J.L. Population biology of plants. London: Academic Press, 1977. 
KAUFFMAN, J.B. Survival by sprouting following fire in tropical forest of the Eastern Amazon. Biotropica, Kansas, v. 23, p.219-224, 1991.

KOTANEN, P.M. Effects of gap area and shape on recolonization by grassland plants with differing reproductive strategies. Canadian Journal of Botany, Ottawa, v. 75, p.352-361, 1997.

MARTINS, S.V.; BARROS, N.F.; SAMPAIO, O.B. et al. Liberação e lixiviação de nutrientes pela queima da manta orgânica de três coberturas vegetais. Revista Árvore, Viçosa, v.19, n.2, p.149-156, 1995.

MIRANDA, M.I.; KLINK, C.A. Colonização de campo sujo de Cerrado com diferentes regimes de queima pela graminea Echinolaena inflexa (Poaceae). In: MIRANDA, H.S.; SAITO, C.H.; DIAS, B.F.S. (Eds.). Impactos de queimadas em áreas de Cerrado e de Restinga. Brasília: Universidade de Brasília, 1996a. p.46-52.

MIRANDA, M.I.; KLINK, C.A. Influência do fogo na alocação de biomassa de Echinolaena inflexa em duas áreas de Campo sujo de Cerrado. In: MIRANDA, H.S.; SAITO, C.H. \& DIAS, B.F.S. (Eds.). Impactos de queimadas em áreas de Cerrado e de Restinga. Brasília: Universidade de Brasília, 1996b. p.37-45.

MUELLER-DOMBOIS, D.; ELLENBERG, H. Aims and methods of vegetation ecology. New York: John Wiley \& Sons, 1974.

MUTCH, R.W.; COOK, W.A. Restoring fire to ecosystems: methods vary with land management goals. In: HARDY, C.C.; ARNO, S.F. (Eds.). The use of fire in forest restoration. Ogden: U.S. Department of Agriculture, Forest Service, 1996. p.9-11.

NASCIMENTO, M.T. Rapid resprouting following fire in juveniles of Metrodorea pubescens (Rutaceae) in a Mesophytic forest in Central Brazil. Ciência e Cultura, Brasília, v.48, p.182-183, 1996.

OLDEMAN, R.A.A. Tropical rainforest, architecture, sylvigenesis and diversity. In: SUTTON, S.L.; WHITMORE, T.C.; CHADWICK, A.C. Tropical rainforest: ecology and management. Oxford: Blackwell Scientific, 1983. p.139-150.

PAUSAS, J.G.; CARBÓ, E.; CATURLA, R.N. et al. Post-fire regeneration patterns in the eastern Iberian Peninsula. Acta Oecologica, Paris, v.20, n.5, p.499-508, 1999.

PENHA, A.S. Propagação vegetativa de espécies arbóreas a partir de raízes gemíferas: representatividade na estrutura fitossociológica e descrição dos padrões de rebrota de uma comunidade florestal, Campinas, São Paulo. 1998. 114p. Dissertação (Mestrado em Biologia Vegetal) - Universidade Estadual de Campinas, Campinas.

RAMOS-NETO, M.B.; PINHEIRO-MACHADO, C. O capim-flecha (Tristachya leiostachya Ness.) e sua importância na dinâmica do fogo no Parque Nacional das Emas. In: MIRANDA, H.S.; SAITO, C.H.; DIAS; B.F.S. (Eds.). Impactos de queimadas em áreas de Cerrado e de Restinga. Brasília: Universidade de Brasília, 1996. p.68-75.

RONDON NETO, R.M. Estudo da regeneração natural e aspectos silviculturais de uma clareira de formação antrópica. 1999. Dissertação (Mestrado em Engenharia Florestal) - Universidade Federal de Lavras, Lavras.

ROSA, C.M.M. Recuperação pós-fogo do estrato rasteiro de um Campo Sujo de Cerrado. 1990. Dissertação (Mestrado) - Universidade de Brasília, Brasília.

SANCHES, P.A. Suelos del tropico: característica y manejo. San José: IICA, 1981.

UHL, C.; JORDAN, C.; CLARK, K. et al. Early plant succession afer cutting and burning in the upper rio Negro region of the Amazon basin. Journal of Ecology, Oxford, v.69, p.631-649, 1981.

UHL, C.; CLARK, K.; CLARK, H. et al. Successional patterns associated with slash-and-burn agriculture in the upper Rio Negro region of the Amazon basin. Biotropica, Kansas, v.14, p.249-254, 1982.

ZAHAWI, R.A.; AUGSPURGER, C.K. Early plant succession in abandoned pastures in Ecuador. Biotropica, Kansas, v.31, n.4, p.540-552, 1999. 
Martins, S.B. et al.

Ciência Florestal, v. 12, n. 1, 2002 